\title{
Taste aversion proneness: A modulator of conditioned consummatory aversions in rats
}

\author{
RALPH L. ELKINS \\ Veterans Administration Medical Center, Augusta, Georgia 30910 \\ and \\ STEPHEN H. HOBBS \\ Augusta College, Augusta, Georgia 30904
}

\begin{abstract}
Sprague-Dawley-derived rats were sequentially conditioned to avoid both anise and saccharinflavored solutions with counterbalanced orders of flavor exposures prior to cyclophosphamideinduced illness. The amounts of saccharin and anise solutions consumed during separate postconditioning single-bottle tests were correlated to determine if aversion strength was consistent across flavors. The saccharin-anise sequence revealed a positive and significant correlation, supporting the hypothesis that conditioned flavor aversions are modulated by some intrasubject factor or factors, a tendency designated as taste aversion proneness. However, a correlational coefficient of marginal significance was obtained from the anise-saccharin sequence, leaving unanswered questions concerning the salience and generality of taste aversion proneness. Despite this limitation, taste aversion proneness is viewed as having important applied implications for aversion therapy approaches to the treatment of human alcoholism.
\end{abstract}

Studies of conditioned consummatory aversions have generated in excess of 1,100 research reports, the overwhelming majority of which have been taste aversion (TA) studies that have appeared during the last decade (cf. Riley \& Clarke, 1977; Riley, Richard, \& Sickel, Note 1). Numerous species readily acquire TAs (baitshyness responses) when flavors are appropriately paired with gastrointestinal distress arising as a consequence of accidental food poisoning, rotational stimulation, radiation exposure, or drug-induced illness. Garcia's (1971) anecdotal evidence that such aversions are common in man is supported by the survey data of Garb and Stunkard (1974) and experimental data from cancer chemotherapy subjects of Bernstein (1978). Additional striking examples of human aversions may be found in the alcoholism treatment literature, which contains numerous reports of aversion formation when actual or symbolic alcoholic beverages were paired with druginduced or verbally induced nausea (Baker \& Cannon, 1979; Elkins, 1975, 1980; Lemere \& Voegtlin, 1950).

The salience of TA formation is well illustrated in rats by their acquisition of long-lasting aversions following a single pairing of a novel flavor with a relatively low dose of an illness-inducing drug and, additionally, by the finding that even highly familiar and relatively bland tap water can be rendered aversive by repeated conditioning

Reprint requests should be addressed to Psychology Research (116BU) Veterans Administration Medical Center, Augusta, Georgia 30910. trials and discrimination training (Elkins, 1973, 1974). Other evidence of the robust nature of TA learning is provided by reports that, unlike most conditioned associations, TAs can be established even when the CS flavor precedes the onset of illness by extended temporal intervals ranging up to as much as $24 \mathrm{~h}$ (Etscorn \& Stephens, 1973). Such aversions have obvious survival value for species that are exposed to toxic consummables and, from the perspective of Seligman (1970), have evolved as "highly prepared" associative adjustments.

Although studies of TA phenomena abound in the recent literature, individual differences in aversion acquisition and retention have received little systematic attention. In an early clinical reference, Voegtlin (1947) reported that some alcoholics subjected to chemical aversion therapy reacted normally to emetic drugs but failed to develop conditioned nausea responses. Noting this, Vogel (1960, p. 275) suggested that "individual differences in conditioning the autonomic nausea response" influence the effectiveness of chemical aversion therapy. Results similar to those of Voegtlin (1947) have also been observed by Elkins $(1975,1980)$. While studying covert sensitization, an aversion therapy in which illness is induced by verbal suggestion, Elkins found that most alcoholics who displayed genuine nausea also developed conditoned alcohol aversions. However, some subjects who consistently displayed behavioral and physiological signs of acute distress failed to condition after many trials. This differential conditionability may be of considerable clinical importance, as indicated by a significantly extended period of mean abstinence for success- 
fully conditioned subjects ( 13.7 months), as opposed to that of consistent nausea responders who failed to condition (4.5 months) and to that of treatment dropouts (5.2 months).

The first experimental reference to individual differences in TAs in nonhumans was apparently made by Garcia, Ervin, and Koelling (1967). Using cyclophosphamide as a conditioning agent, saccharin as the target flavor, two rats per dose level, and a relatively insensitive single-bottle extinction procedure that pitted aversion maintenance against fluid deprivation, Garcia et al. concluded that "an examination of individual differences reveals a marked uniformity of response; hence it appears that 'bait shyness' might provide an excellent test for toxic illness requiring only small groups of animals" (1967, p. 245). However, Elkins (1973) performed a systematic replication of their procedure using larger subject groups and found considerable withingroup variability in aversion acquisition. Elkins also compared the Garcia et al. (1967) single-bottle measurement technique with a two-bottle extinction test designed to minimize deprivation effects by providing ad-lib access to both the CS flavor and plain tap water. Subjects conditioned with low doses of cyclophosphamide and evaluated with this two-bottle test displayed marked individual differences in both initial aversion magnitude and resistance to extinction. Similar individual differences were reported following a study of conditioned water aversions (Elkins, 1974) and have been consistently observed within our laboratories when aversion induction has been based on moderate or light doses of illness-inducing drugs.

These results show that rats subjected to equal drug doses can display considerable variability in aversion strength, but they are inconclusive relative to the nature of such differences. For instance, the individual differences observed by Elkins $(1973,1974)$ might reflect a "TA proneness" trait, a tendency for intrasubject factors to modulate the efficiency of aversion formation and maintenance. However, they could also arise from some other factor, such as differences associated with the vagaries of intraperitoneal injection. This experiment was therefore designed as a more direct test of the TA proneness hypothesis. If intrasubject factors constitute a significant source of variation in flavor aversion conditioning, then individual subjects should show some consistency in the degree to which they acquire aversions to different flavors. We therefore decided to sequentially condition the same rats to avoid two distinct flavors, to determine the degree to which individual acquisition of these different aversions was correlated, and to accept a significant positive correlation as support for the hypothesis that TA acquisition is modulated by individual differences in TA proneness.

\section{METHOD}

\section{Subjects}

Twenty-four Sprague-Dawley-derived male rats weighing from 400 to $500 \mathrm{~g}$ and experimentally naive at the beginning of the study served as subjects. All animals were individually housed in standard wire cages and adapted to the laboratory for at least 2 weeks prior to any experimentation. They were maintained under a natural light-dark cycle and had ad-lib access to Purina Laboratory Rat Chow during both adaptation and experimental periods.

\section{Procedure}

Subjects were randomly assigned to one of four groups, two experimental groups containing eight subjects each and two control groups of four subjects each. Experimental subjects were sequentially conditioned to avoid both an anise-flavored solution (.08\% Sauers' anise extract in water) and a saccharin solution (.05\% sodium saccharin in water). One experimental group and one control group were exposed to anise in the first conditioning session and to saccharin in the second session, and this order of flavor presentation was reversed for the other two groups. Conditioning episodes were separated by 15 days and were conducted between 1400 and $1500 \mathrm{~h}$ Eastern Standard time with subjects under 24-h fluid deprivation. During each conditioning session, subjects were given $15 \mathrm{~min}$ of home-cage access to either the anise or the saccharin flavor. Five minutes after flavor removal, experimental subjects received a $25-\mathrm{mg} / \mathrm{kg}$ intraperitoneal cyclophosphamide injection (Cytoxan, ${ }^{\circledR}$ Mead Johnson Laboratories), and control subjects received an equivalent volume of normal saline. The same investigator administered all injections, using a plastic rodent-restraining device to minimize differential handling effects. He was also unaware of the performance of individual subjects, relative to the first aversion test, when giving injections following the second conditioning session. In an effort to minimize the negative effects of high fluid deprivation on aversion detection, subjects were given $1 \mathrm{~h}$ of access to tap water $6 \mathrm{~h}$ after injection.

TA formation was assessed with a single-bottle test procedure in which subjects were given $15 \mathrm{~min}$ of home-cage access to the target flavor $24 \mathrm{~h}$ after each conditioning session. Bottles were filled prior to and weighed both before and after testing. Fluid ingestion was computed from these pre/post weights, and aversion development was evaluated by comparing fluid consumption of experimental and control subjects with Mann-Whitney $\mathrm{U}$ tests. The tendency of individual subjects to show a trait of bait-shyness proneness was assessed through a Pearson productmoment correlation of experimental subjects' test consumption of the anise and saccharin solutions.

\section{RESULTS AND DISCUSSION}

As seen in Table 1, control subjects invariably generated substantially higher mean and median consumption scores than did experimental subjects. This table also shows that there was no individual overlap in amount consumed by experimental subjects as compared to controls. This pattern produced Mann-Whitney

Table 1

Mean, Median, and Range of Saccharin and Anise Consumption (in Milliliters) During the 15-Minute Single-Bottle Postconditioning Tests

\begin{tabular}{lccccccc}
\hline & \multicolumn{3}{c}{ Experimental } & \multicolumn{3}{c}{ Control } \\
\cline { 2 - 3 } \cline { 5 - 7 } \cline { 5 - 6 } & Mean Median & Range & Mean Median & Range \\
\hline Saccharin & 3.0 & .9 & $.3-8.0$ & 15.5 & 13.3 & $9.0-20.2$ \\
Anise & 1.7 & .5 & $.0-5.2$ & 11.6 & 9.5 & $8.4-14.9$ \\
& \multicolumn{5}{c}{ Anise-Saccharin Sequence } \\
Anise & 2.6 & 2.0 & $.6-7.2$ & 14.1 & 13.5 & $10.7-17.9$ \\
Saccharin & 2.5 & 1.1 & $.4-7.7$ & 13.3 & 13.4 & $10.5-15.5$ \\
\hline
\end{tabular}


$\mathrm{U}$ values of 0 for each experimental-control comparison and indicated that the experimental groups consumed less than their corresponding controls at the .01 level of statistical significance (Siegel, 1956).

There was an overall correlation of +.54 between postconditioning measures of CS consumption on the two extinction tests, a value significantly greater than 0 ( $p<.025$, one-tailed $)$. This overall correlation dropped to $+.31(\mathrm{p}<.10$, one-tailed) when the anise-saccharin sequence was considered alone and increased to +.90 $(\mathrm{p}<.025$, one-tailed) for the saccharin-anise sequence considered separately. These data are displayed in Figure 1.

Results of the anise-saccharin sequence are somewhat ambiguous, as indicated by a positive correlation coefficient that approximated but failed to satisfy the conventional .05 level of statistical significance. Results of the saccharin-anise sequence appear to support the TA proneness hypothesis. However, another factor relative to the saccharin-anise sequence must be considered before such a conclusion is justified. Specifically, the magnitude of the saccharin aversion was positively correlated $(\mathrm{r}=+.70)$ with anise consumption during the second conditioning session, and this CS consumption was similarly correlated $(r=+.64)$ with magnitude of the subsequent anise aversion. The +.90 correlation between aversion magnitudes from the saccharin-anise sequence could, therefore, be a function of this mutual positive correlation with anise consumption during the second conditioning session. A partial correlation was, therefore, computed for the saccharin-anise sequence to determine the degree of relationship between the first and second conditionings independent of the effects of

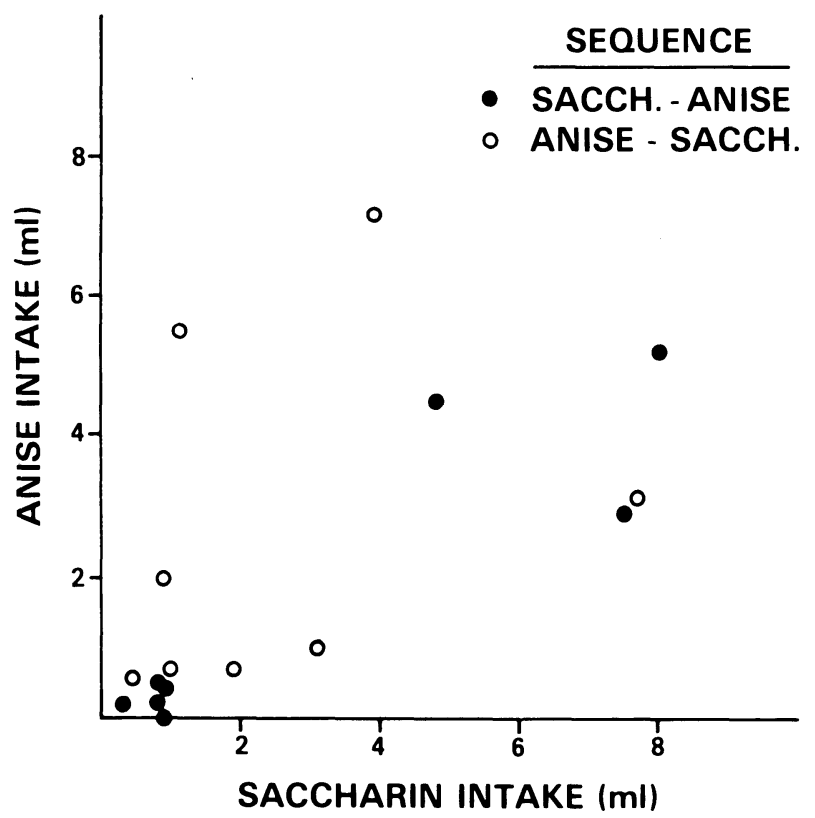

Figure 1.Anise- and saccharin-solution intake during postconditioning tests of aversion strength.
CS consumption during the second (anise) conditioning trial. This parital $\mathrm{r}$ was found to be +.82 , a value significantly greater than $0(p<.05$, one-tailed $)$, a result supporting the TA proneness hypothesis. A corresponding partial correlation was not computed for the anisesaccharin sequence because the $\mathrm{r}$ of +.31 , relating anise test consumption to saccharin test consumption, failed to reach conventional significance levels. Such an analysis was also contraindicated by the relationship between Session 1 consumption and CS acceptance during conditioning Session 2, which markedly violated the linearity assumption basic to the partial correlational procedure (McNemar, 1962).

Results of the saccharin-anise sequence support the TA proneness hypothesis, but the finding that order of aversion establishment modulates the degree to which aversion strengths are correlated raises additional questions. If these order effects prove to be reliable, their bases may be clarified by analyses of palatability factors. Quantitative data relative to the palatability of the present saccharin and anise solution are unavailable. However, unpublished results of two-bottle preference tests conducted in our laboratories show that rats of this strain and age strongly prefer the saccharin solution to tap water, but they also prefer tap water to the anise solution. Thus, differential palatability of solutions may have contributed to order effects, a possibility that could be explored in studies using distinctive flavors of equivalent acceptability.

Present results are consistent with the Elkins (1973) suggestion that both rats and humans may have evolved flavor-toxicosis associative mechanisms displaying intraspecific variability in the efficiency of aversion acquisition. However, differential efficiency of central associative mechanisms is only one of several possible explanations. For example, TA proneness might reflect individual differences in sensitivity, either to the CS stimuli or to the internal manifestations of cyclophosphamideinduced illness. The former of these two possibilities is discussed by Horowitz and Whitney (1975), who have observed marked differences in the ease with which two genetically different strains of inbred mice acquire conditioned saccharin aversions based on ethanol injections. Barker, Suarez, and Gray's (1974) report of individual differences in observable manifestations of illness in rats given $75-\mathrm{mg} / \mathrm{kg}$ intraperitoneal cyclophosphamide injections is relevant to the latter alternative. Barker and his colleagues reported no observable illness in 30\% to $40 \%$ of their subjects and varying degrees of distress in the others, as indicated by response patterns ranging from decreased motor activity $(50 \%$ of the subjects) to acute locomotor disability (10\%$15 \%$ of the subjects). However, Barker et al. (1974) found no relationship between these overt signs of toxicosis and subsequent conditioning. While these observations question differential sickness as a basis for TA proneness, Barker et al. (1974) used much higher 
cyclophosphamide doses than those of the present study. The possibility exists that our findings were influenced by individual differences in illness that are not readily detectable by gross observational techniques. Much research will be required to determine the mechanism or mechanisms underlying TA proneness.

Selective breeding of Sprague-Dawley-derived rats, initiated at the completion of the present experiment, is providing strong additional support for the TA proneness hypothesis. Strong and weak acquirers of a cyclophosphamide-induced saccharin aversion have been selectively bred over seven generations. Significant and progressive strain-separation has been observed to a degree that is suggestive of considerable genetic control of individual differences in TA conditionability (Elkins, Note 2). Heritability will be quantified after asymptomatic strain separation has been achieved, and studies of the physiological bases of the attained differences will be initiated. It is hoped that these efforts will clarify the basic nature of TA learning and will facilitate and expedite efforts to maximize the efficiency of aversion therapy approaches to alcoholism treatment.

\section{REFERENCE NOTES}

1. Riley, A. L., Richard, K. D., \& Sickel, J. L. Conditioned taste aversions: $A$ bibliography. Printout of a computerized bibliography as of September 1981.

2. Elkins, R. L. Differential learning of illness-induced taste aversions and shock-motivated environmental avoidances by rats selectively bred for taste aversion proneness or resistance to taste aversion acquisition. Paper presented at the annual meeting of the Southeastern Psychological Association, New Orleans, 1982.

\section{REFERENCES}

Baxer, T., \& Cannon, D. S. Taste aversion therapy with alcoholics: Techniques and evidence of a conditioned response. Behavior Research and Therapy, 1979, 17, 229-242.

Barker, L. M., Suarez, E. M., \& Gray, D. Backward conditioning of taste aversions in rats using cyclophosphamide as the US. Physiological Psychology, 1974, 2, 117-119.

Bernste in, I. L. Learned taste aversions in children receiving chemotherapy. Science, 1978, 200, 1302-1303.
Elkins, R. L. Individual differences in bait shyness: Effects of drug dose and measurement technique. Psychological Record, 1973, 23, 349-358.

Elkins, R. L. Conditioned flavor aversions to familiar tap water in rats: An adjustment with implications for aversion therapy treatment of alcoholism and obesity. Journal of Abnormal Psychology, 1974, 83, 411-417.

Elkins, R. L. Aversion therapy for alcoholics: Chemical, electrical or verbal-imaginary. International Journal of the Addictions, 1975, 10, 257-309.

Elkins, R. L. Covert sensitization treatment of alcoholism: Contributions of successful conditioning to subsequent abstinence maintenance. Addictive Behaviors, 1980, 5, 67-89.

Etscorn, F., \& Stephens, R. Establishment of conditioned taste aversions with a 24-hour CS-US interval. Physiological Psychology, 1973, 1, 251-253.

Garb, J. L., \& Stunkard, A. J. Taste aversions in man. American Journal of Psychiatry, 1974, 131, 1204-1207.

Garcin, J. The faddy rat and us. New Scientist and Science Journal, February 1971, pp. 254-256.

Garcia, J., Ervin, F., \& Koelling, R. Bait-shyness: A test for toxicity with $\mathbf{N}=2$. Psychonomic Science, 1967, 7, 245246.

Horowitz, G. P., \& Whitney, G. Alcohol-induced conditioned aversion: Genotypic specificity in mice (Mus musculus). Journal of Comparative and Physiological Psychology, 1975, 89, 340346.

Lemere, F., \& Voegtin, W. L. An evaluation of the aversion treatment of alcoholism. Quarterly Journal of Studies on Alcohol, 1950, 11, 199-204.

McNemar, Q. Psychological statistics. New York: Wiley, 1962.

Riley, A. L., \& Clarke, C. M. Conditioned taste aversions: A bibliography. In L. M. Barker, M. R. Best, \& M. Domjan (Eds.), Learning mechanisms in food selection. Waco, Tex: Baylor University Press, 1977.

Seligman, M. E. P. On the generality of the laws of learning. Psychological Review, 1970, 77, 406-418.

Siegel, S. Nonparametric statistics for the behavioral sciences. New York: McGraw-Hill, 1956.

Voegtuin, W. L. Conditioned reflex therapy of chronic alcoholism. Ten years' experience with the method. Rocky Mountain Medical Journal, 1947, 44, 807-812.

VOGEL, M. D. The relation of personality factors to GSR conditioning of alcoholics: An exploratory study. Canadian Journal of Psychology, 1960, 14, 275-280.

(Received for publication October 7, 1982.) 